\title{
Causes of nutrition deficit during immediate postoperative period after free flap surgery for cancer of the head and neck
}

\author{
Juho Nurkkala ${ }^{1}$ D $\cdot$ Sanna Lahtinen ${ }^{1} \cdot$ Timo Kaakinen $^{1} \cdot$ Merja Vakkala $^{1} \cdot$ Janne Liisanantti $^{1}$
}

Received: 19 May 2020 / Accepted: 8 July 2020 / Published online: 14 July 2020

(c) The Author(s) 2020

\begin{abstract}
Purpose The aim of the present of study was to examine nutrition deficit during the immediate postoperative in-hospital period following free flap surgery for cancer of the head and neck (HNC). Underfeeding and malnutrition are known to be associated with impaired short- and long-time recovery after major surgery.

Methods This single-center retrospective cohort study included $218 \mathrm{HNC}$ patients who underwent free flap surgery in Oulu University Hospital, Finland between the years 2008 and 2018. Nutrition delivery methods, the adequacy of nutrition and complication rates were evaluated during the first 10 postoperative days.

Results A total of $131(60.1 \%)$ patients reached nutritional adequacy of $60 \%$ of calculated individual demand during the follow-up period. According to multivariate analysis, nutrition inadequacy was associated with higher ideal body weight (OR 1.11 [1.04-1.20]), whereas adequate nutrition was associated with higher number of days with oral food intake (OR 0.79 [0.67-0.93]).

Conclusion Inadequate nutrition is common after HNC free flap surgery. The present results suggest that more adequate nutrition delivery might be obtained by the early initiation of oral food intake and close monitoring of nutrition support.
\end{abstract}

Keywords Head and neck cancer · Free flap · Nutrition · Nutrition support · Enteral nutrition · Parenteral nutrition

\section{Introduction}

Among patients undergoing major surgical operations, preoperative malnutrition and postoperative underfeeding are recognized risk factors for postoperative complications such as prolonged hospital length of stay (LOS), higher incidence of infectious complications, impaired physical recovery and increased morbidity [1]. It is essential to maintain adequate nutrition after surgical procedures as it improves wound healing. Underfeeding is known to impede normal wound recovery process and is related to wound infections and impaired wound tensile strength [2]. Previous studies contemplating the adequacy of postoperative nutrition have

Sanna Lahtinen and Timo Kaakinen contributed equally to this work.

Juho Nurkkala

juho.nurkkala@student.oulu.fi

1 Department of Anesthesiology, Medical Research Centre and Research Group of Surgery, Anesthesia and Intensive Care, University of Oulu, Oulu University Hospital, P.O. Box 21, 90029 Oulu, Finland been conducted mainly after gastrointestinal, orthopedic and thoracic surgery [1,3-6]. There is a lack of studies focusing on the nutrition adequacy after free flap reconstruction of the tumor resections for the cancer of the head and neck (HNC).

Median hospital LOS after free flap surgery for HNC is more than week [7-9]. In-hospital nutrition plays a significant role in the early recovery in this patient group [10]. Although HNC free flap surgery may have a substantial impact on normal eating, chewing and swallowing, there is a lack of studies contemplating the adequacy of nutrition during the immediate postoperative period. Studies concerning the postoperative nutrition of hospitalized HNC patients have mostly been focusing on the preceding prevalence of malnutrition $[11,12]$ and optimal initiation of oral food intake such as in laryngectomized patients in terms of pharyngocutaneus fistula formation [13-15].

The aim of the present study was to evaluate the adequacy of nutrition after free flap surgery for HNC during the immediate in-hospital recovery and to discover factors associated with inadequate nutrition delivery. 


\section{Materials and methods}

This retrospective longitudinal cohort study was conducted in Oulu University Hospital in Oulu (OUH), Finland. The study protocol was accepted by the hospital administration (208/2015 and 239/2016). Following the local policy, no statement from the ethics committee was obtained due to the retrospective study design.

\section{Patients}

The study population consisted of all patients undergone HNC free flap surgery in Oulu University Hospital between the years 2008 and 2018. During the study period, a total of 247 free flap operations were performed. 29 cases were excluded from the analysis due to incomplete data leaving a total of 218 cases. Since the median hospital length of stay (LOS) is more than 7 days in this patient group, we included the first 10 postoperative in-hospital days in the analysis [7, 9].

\section{Nutrition delivery}

The ideal body weight (IBW) was calculated for each patient using the Devine formula for men and the Robinson formula for women [11]. The energy demand during the study period was calculated individually for each patient using the ideal weight and the estimate of $30 \mathrm{kcal} / \mathrm{kg} /$ day, which was derived from both the ESPEN guidelines for surgical patients and from the national nutritional guidelines of the UK for HNC patients [1, 10]. Current literature is lacking studies focusing on the adequacy of postoperative nutrition of HNC free flap patients. We selected a cut-off value of $60 \%$ of calculated energy demand to mark adequate nutrition in the present study. The cut-off value of $60 \%$ has been used in an intensive care setting to depict the difference between satisfactory nutrition and malnutrition $[16,17]$. The screening of malnutrition was performed with a modified nutritionrelated index (NRI) presented by Parhar et al. [11]. NRI was calculated by the following formula: $[1.519 \times$ serum $\operatorname{albumin}(\mathrm{g} / \mathrm{l})]+[41.7 \times(\operatorname{mass} / \mathrm{IBW}(\mathrm{kg}))]$. Patients with NRI less than 97.5 were considered as preoperatively malnourished. The total amount of consumed intravenous 5\% dextrose, PN (parenteral nutrition), EN (enteral nutrition) and oral food intake was calculated by adding all delivered calories during the follow-up time and dividing the sum by the number of follow-up days. Oral food intake calories were calculated from the patient records by inspecting the daily food consumption in milliliters and approximating the daily content of calories based on the average hospital diet (1800 kcal/day in OUH). In the present study, the nutrition support consisted 5\% dextrose, PN and EN calories. The loss of appetite, gastric pain and nausea were recorded when the patient refused oral or enteral nutrition at least once due to these reasons during the follow-up time. The study group with $<60 \%$ of calculated energy demand is described below as "study group low", and the study group with $>60 \%$ of calculated energy demand is described below as "study group adequate" for clarity issues.

\section{Postoperative complications}

Since perioperative underfeeding and malnutrition are known to have an impact on postoperative complications and outcome [1], we aimed to evaluate rates of complications in the present study. Complications were classified as medical and surgical and were recorded as in our previous study [18].

\section{Statistical analysis}

Statistical analysis was performed with IBM SPSS Statistics 25 software (IBM SPSS Statistics for Windows, Version 25.0, Armonk, NY, USA). Categorical data are expressed as numbers $(n)$ and percentages $(\%)$ whereas continuous data are expressed as medians and 25 th-75th percentiles [25th-75th PCT]. Categorical data were tested using the Pearson's chi square and the continuous variables were tested using the non-parametric Mann-Whitney test. Twotailed $P$ value was considered statistically significant when it was below 0.05 . The logistic regression analysis was used to calculate OR for not reaching the $60 \%$ nutrition adequacy cut-off value. Continuous and categorial variables with univariate significance $<0.1$ as well as age and gender were included one by one using the enter method. The factors with $P$ value $<0.05$ were kept in the model, as well as those with significant impact on the log-likelihood function.

\section{Results}

There were a total of $131(60.1 \%)$ patients who achieved the cut-off value of nutritional adequacy in the study group adequate. Patients in the study group low were more often males, had a higher IBW and a higher rate of smoking and alcohol abuse and they had a longer hospital LOS (Table 1). Among the 115 patients with larynx or oral cavity/tongue tumor, a total of 54 (46.9\%) were in group low in contrast to 33 of $103(32.0 \%, P=0.04)$ in other tumors.

The patients in the study group adequate had a lower calculated daily energy demand, higher administration of enteral calories, earlier initiation of oral food intake and a higher number of days with oral food intake. Patients in the study group low had more often gastric pain (Table 2). In the study group, adequate oral food intake increased overall 
Table 1 Patient demographics

\begin{tabular}{llll}
\hline & Group adequate $(N=131)$ & Group low $(N=87)$ & $P$ value \\
\hline Male gender & $56(41.2)$ & $65(74.7)$ & $<0.001$ \\
Age, years & $67.0[57.0-75.0]$ & $63.0[57.0-73.0]$ & 0.172 \\
Ideal body weight $(\mathrm{kg})$ & $59.5[52.3-66.8]$ & $68.7[59.6-75.0]$ & $<0.001$ \\
BMI & $24.4[21.6-27.9]$ & $23.9[20.0-27.3]$ & 0.147 \\
Preoperatively malnourished & $20(15.3)$ & $18(20.7)$ & 0.459 \\
Smoking & $49(37.4)$ & $45(51.7)$ & 0.050 \\
Alcohol & $31(23.7)$ & $34(39.1)$ & 0.021 \\
Tumor & & & \\
Oral cavity/tongue & $53(40.5)$ & $40(46.0)$ & 0.072 \\
Maxilla & $13(9.9)$ & $10(11.5)$ & \\
Mandible & $24(18.3)$ & $12(13.8)$ & \\
Larynx & $8(6.1)$ & $14(16.1)$ & \\
Skin melanoma & $12(9.2)$ & $6(6.9)$ & \\
Buccal mucosa & $15(11.5)$ & $5(5.7)$ & \\
Parotid gland & $5(3.8)$ & $0(0.0)$ & 0.638 \\
Lymphoma & $1(0.8)$ & $0(0.0)$ & 0.029 \\
Stage T1-2 & $33(25.2)$ & $26(29.9)$ & $48(55.2)$ \\
Stage T3-4 & $73(55.7)$ & $13[8-16]$ & \\
Hospital LOS (days) & $10[8-14]$ & 109 & \\
\hline
\end{tabular}

$B M I$ body mass index, LOS length of stay. Values are numbers (percentage) or medians [25th-75th percentiles].

Table 2 Nutritional characteristics of the patients receiving either $\geq 60 \%$ or $<60 \%$ of calculated energy demand

\begin{tabular}{lllr}
\hline & $\begin{array}{l}\text { Group adequate } \\
(N=131)\end{array}$ & Group low $(N=87)$ & $P$ value \\
\hline Calculated daily energy demand $(\mathrm{kcal} / \mathrm{d})$ & $1784[1569-2005]$ & $2060[1788-2250]$ & $<0.001$ \\
Administered daily 5\% dextrose $(\mathrm{kcal})$ & $97[56-140]$ & $91[43-154]$ & 0.773 \\
Administered daily PN $(\mathrm{kcal})$ & $200[97-280]$ & $174[70-288]$ & 0.501 \\
Administered daily EN $(\mathrm{kcal})$ & $344[115-630]$ & $374[178-573]$ & 0.822 \\
Administered daily oral food intake (kcal) & $675[315-900]$ & $180[0-550]$ & $<0.001$ \\
First day of oral food intake & $5[3-7]$ & $6[5-8]$ & $<0.001$ \\
Days of oral food intake & $6[4-8]$ & $5[3-6]$ & 0.001 \\
Loss of appetite & $21(16.0)$ & $14(16.1)$ & $>0.9$ \\
Gastric pain & $12(9.2)$ & $19(21.8)$ & 0.010 \\
Nausea & $30(22.9)$ & $16(18.4)$ & 0.499 \\
\hline
\end{tabular}

$P N$ parenteral nutrition, $E N$ enteral nutrition. Values are numbers (percentage) or medians [25th-75th percentiles] nutrition adequacy. Nutrition adequacy improved during first seven days but started to decrease as the number of discharged patients increased (Fig. 1).

In the surgical characteristics, the patients in the study group low had more often tracheostomy and bilateral neck dissection (Table 3).

The patients in the study group low had more often postoperative surgical site infections, although there was no difference between the groups in the onset of infection (3 [2-10] vs 6 [2-11], $P=0.488$ ). There was no difference in the incidence of partial or total loss of flap but the patients in the study group low suffered a loss of flap earlier than in the study group adequate (3 [2-20] vs 10 [6-13], $P=0.050$ ). Overall postoperative surgical complications were more frequent among patients in the study group low. There was no difference in the incidence or distribution of medical complications between the study groups (Table 4).

In the logistic regression analysis, a higher IBW was associated with a failure to reach nutritional targets whereas a higher number of days with oral food intake was associated with a decreased OR for impaired nutrition delivery (Table 5). 


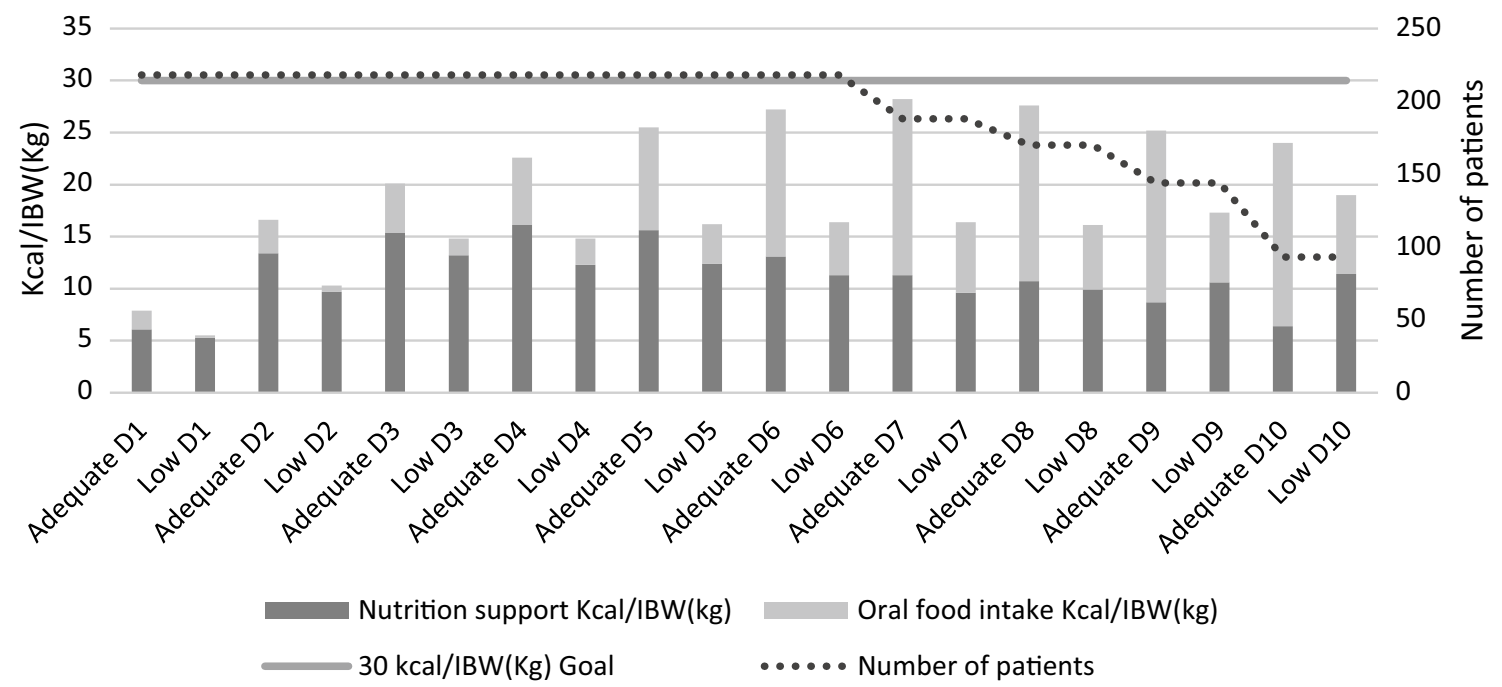

Fig. 1 The mean daily delivery of nutrition support and oral food intake. The values are presented for the group adequate and for the group low as the administered calories divided by ideal body weight (IBW in kg's)

Table 3 Surgical features of the patients receiving either $\geq 60 \%$ or $<60 \%$ of calculated energy demand

\begin{tabular}{llll}
\hline & Group adequate $(N=131)$ & Group low $(N=87)$ & $P$ value \\
\hline Tracheostomy & $96(73.3)$ & $74(85.1)$ & 0.046 \\
Bilateral neck dissection & $14(10.7)$ & $19(21.8)$ & 0.016 \\
Unilateral neck dissection & $87(66.4)$ & $42(48.3)$ & \\
No neck dissection & $30(22.9)$ & $26(29.9)$ & \\
Flap type & & & \\
RFA & $47(35.9)$ & $30(34.5)$ & \\
ALT & $46(35.1)$ & $23(26.4)$ & \\
LD & $3(2.3)$ & $1(1.1)$ & \\
Scapula & $6(4.6)$ & $3(3.4)$ & $15(17.2)$ \\
Fibula & $18(13.7)$ & $7(8.0)$ & 0.474 \\
Lateral arm & $6(4.6)$ & $7(8.0)$ & 0.514 \\
Other & $5(3.8)$ & $550[350-950]$ & 0.752 \\
Intraoperative blood loss (ml) & $600[350-1000]$ & $5890[4400-7330]$ & \\
Intraoperative infused fluids (ml) & $5910[4450-7870]$ & $129[42-336]$ & 0.259 \\
Intraoperative duration of MAP $<65$ (min) & $148[50-256]$ & $541[440-621]$ & \\
Length of surgery (min) & $495[426-620]$ & &
\end{tabular}

$R F A$ radial forearm flap, $A L T$ anterolateral thigh flap, $L D$ latissimus dorsi flap, $M A P$ mean arterial pressure. Values are numbers (percentage) or medians [25th-75th percentiles]

\section{Discussion}

For our understanding, this is the first study evaluating postoperative in hospital nutrition adequacy after HNC free flap surgery. The main finding of the present study was that inadequate nutrition delivery was common in patients with HNC undergoing free flap surgery. Approximately, $40 \%$ of patients did not achieve the cut-off value of $60 \%$ of calculated energy demand during the first 10 postoperative days. Our results suggests that an early initiation of oral food intake significantly improved nutrition delivery. Enteral or parenteral nutrition support alone often did not provide adequate nutrition delivery for patients without oral intake. Patients with lower IBW were more likely to reach a sufficient level of calories when compared patients with higher IBW indicating that individual demand of energy was not always taken into consideration. Patients whose operation affected oral cavity or larynx had lower nutritional status than other subgroups. Patients with postoperative surgical complications were associated with 
Table 4 Postoperative complications

\begin{tabular}{lllc}
\hline & Group adequate $(N=131)$ & Group low $(N=87)$ & $P$ value \\
\hline Surgical complications & $54(41.2)$ & $49(56.3)$ & 0.037 \\
Surgical site infection & $21(17.3)$ & $29(30.7)$ & 0.003 \\
Onset of infection (d) & $6[2-11]$ & $3[2-10]$ & 0.488 \\
Surgical site hematoma & $23(17.6)$ & $16(18.4)$ & $>0.9$ \\
Onset of hematoma (d) & $2[1-5]$ & $4[2-7]$ & 0.128 \\
Reoperation & $36(27.5)$ & $33(37.9)$ & 0.104 \\
Onset of reoperation (d) & $5[1-9]$ & $2[1-9]$ & 0.875 \\
Partial or total flap loss & $9(6.9)$ & $9(10.3)$ & 0.452 \\
Onset of partial or total flap loss (d) & $10[6-13]$ & $3[2-20]$ & 0.050 \\
Medical complications & $36(25.2)$ & $24(26.4)$ & 0.9 \\
Pneumonia & $21(16.0)$ & $18(20.7)$ & 0.471 \\
Onset of pneumonia (d) & $4[2-7]$ & $7[3-10]$ & 0.140 \\
Sepsis & $5(3.8)$ & $2(2.3)$ & 0.705 \\
Sepsis onset days & $2,7,8,8,21$ & 1,13 & 0.370 \\
Pulmonary edema & $9(6.9)$ & $3(3.4)$ & \\
Pulmonary edema onset days & $1,3,4,6,6,7,8,9,18$ & $4,7,9$ & 0.158 \\
Acute myocardial infarction & $0(0.0)$ & $2(2.3)$ & 0.732 \\
Acute myocardial infarction onset days & - & 1,3 & $2(2,3)$ \\
Deep venous thrombosis, pulmonary & $4(3,1)$ & 2,9 & \\
embolism, stroke & $2,3,5,11$ & & \\
Deep venous thrombosis, pulmonary & & & \\
embolism, stroke onset days & & & \\
\hline
\end{tabular}

Values are numbers (percentage) or medians [25th-75th percentiles]. Sepsis, pulmonary edema, acute myocardial infarction and deep venous thrombosis/pulmonary embolism/stroke onset days are reported without percentiles due to small count of cases
Table 5 OR and 95\% confidence intervals for impaired nutrition delivery according to the logistic regression analysis

\begin{tabular}{lll}
\hline & OR $(95 \%$ CI $)$ & $P$ value \\
\hline Male gender & $0.52(0.14-1.84)$ & 0.307 \\
Age & $1.01(0.98-1.04)$ & 0.468 \\
Ideal body weight & $1.11(1.04-1.20)$ & 0.002 \\
Surgical complications & $0.84(0.40-1.76)$ & 0.640 \\
Days with oral food intake & $0.79(0.67-0.93)$ & 0.005 \\
\hline
\end{tabular}

nutrition inadequacy, although the difference was not seen in the logistic regression analysis.

According to our results, an early initiation of oral food intake improved nutrition delivery considerably. Furthermore, oral food intake was a more efficient way to deliver nutrition compared to nutrition support, although calculated caloric demand was never achieved with any nutrition delivery method. Both the ESPEN guidelines and the national nutritional guidelines of the United Kingdom (UK) for HNC patients promote early postoperative oral feeding or nutrition support in this patient group [1, 10]. It is previously known that $\mathrm{HNC}$ patients undergone free flap surgery are rarely able to eat normally immediately after the operation $[1,10]$. Common reasons for impaired oral food intake after HNC free flap surgery are dysphagia and swallowing-related pain $[7,18]$. Due to anatomic and physiological reasons, swallowing may be permanently deteriorated after free flap surgery for HNC [19]. HNC surgery may also cause significant oral cavity or upper gastrointestinal tract swelling during first postoperative days inhibiting oral food intake [1]. It is also reported recently by Lilja et al. that HNC free flap surgery affecting oral cavity may impair taste sensation which affects negatively oral food intake [20]. Moreover, growing HNC tumor may develop upper gastrointestinal tract obstruction which causes difficulties in swallowing and furthermore induces patients to eat less than without the illness [11].

In the past, surgeons used to delay the initiation of oral food intake among HNC patients with free flap surgery affecting the oral cavity for avoiding adverse outcomes in wound healing and reducing the risk of dehiscence. Recent studies have shown that early oral food intake after surgery affecting the oral cavity is safe $[8,21]$. For example, many studies from recent years have shown that early oral food intake does not increase pharyngocutaneus fistula formation. Nowadays early oral food intake is recommended also for patients with total laryngectomy [10, 13-15]. According to the present study, it seems that efforts should be made to initiate oral food intake as soon as possible after the operation to obtain adequate nutrition delivery. However, due to 
reasons mentioned above, oral food is rarely given during the very first postoperative days and in the previous literature "early oral intake after HNC surgery" is generally determined as oral nutrition given before or during the fifth postoperative day $[14,21]$ which in this study was the median oral food initiation day in the study group adequate.

The results of the present study suggest that nutrition support did not provide sufficient intake of calories for patients whose oral food intake was inadequate. Patients unable to eat could benefit from individual nutrition support to enhance their overall nutrition status. In the intensive care setting, it has been reported that nutrition adequacy can be as low as $30 \%$ of calculated demand when feeding is conducted solely by nutrition support [22]. The impact of nutrition support has become more evident in the intensive care by the introduction of nutrition support protocols, inspecting the patient's nutritional status twice a day and by including a routine dietician consultation for every patient [16, 23]. These methods could also be utilized in HNC patients to enhance nutrition support.

The patients with tracheostomy had a greater risk for postoperative undernutrition in the univariate model. The negative effect of tracheostomy to nutrition delivery was an expected finding since the procedure diminishes tolerance to oral food intake which was the most dominant nutrition delivery method in the present study [24]. Bartella et al. reported recently strong statistically significant relation between impaired swallowing function and prolonged timing of decannulation [25]. Moreover, Goetz $\mathrm{C}$ et al. recommended that the duration of temporary tracheostomy in $\mathrm{HNC}$ patients should be as short as possible to reduce associated complications [26]. Considering the nutritional aspect, a similar conclusion can be made based on our results. In the present study, bilateral neck dissection was a risk factor for impaired nutrition adequacy in the univariate model. It has been previously reported that bilateral neck dissection has a negative impact on swallowing function after HNC free flap surgery [19], which might explain the association between a poor nutrition adequacy and bilateral neck dissection.

The occurrence of surgical complications was more frequent among patients with inadequate nutrition delivery. Postoperative surgical site infections were more frequent in patients with impaired nutrition adequacy in the present study. It has been reported that postoperative malnutrition in surgical and intensive care patients predisposes to infectious complications $[1,2,10,16,23]$. On the other hand, it is known that postoperative surgical site infections are a risk factor to reoperations [9], which may predispose to malnutrition caused by preoperative fasting. In the present study, however, there was no difference in the onset of infection between the study groups. There was also no statistically significant difference in the incidence rates of partial or total loss of flaps between the study groups but patients with a lower nutrition adequacy had flap losses in an earlier phase of their recovery. Partial or total loss of flap may cause delay in initiation of oral food intake due to preoperative fasting before reoperations. Also, uncertainty of the healing status of wounds affecting oral cavity may cause surgeon to postpone initiation of oral nutrition. Group adequate had their loss of flaps later which may explain why their nutrition status was higher during present follow-up. No difference was found in other subgroups of surgical or medical complications. Due to the retrospective nature of the study and relatively short follow-up time, a causal relationship between inadequate nutrition delivery and the occurrence of postoperative complications remains obscure.

\section{Clinical significance}

The negative impact of postoperative malnutrition on both short- and long-term recovery of surgical patients has been widely acknowledged and, therefore, pursuing adequate nutrition delivery is desirable $[1,10]$. The results of present study add to the knowledge that reaching sufficient nutrition for $\mathrm{HNC}$ free flap patients postoperatively is beneficial. Based on our results, efforts should be made to obtain sufficient nutrition delivery and focus should be turned to early oral food intake. This could be attained by adequate pain medication and by utilizing consultation of a dietician and a physiotherapist in the immediate recovery phase [10]. For patients whose oral food intake is insufficient, nutrition support delivery could be increased by using specified nutrition protocols and by inspecting the nutritional status twice a day $[1,23]$. A multidisciplinary team (surgeon, physiotherapist, dietician, trained nurse) could be very useful in establishing and enhancing individual nutritional support for patients after HNC free flap surgery as well as after any major surgery [10]. Further research, preferably in a prospective setting, should be conducted to enlighten the possible connection between these proposed means and adequate nutritional delivery among $\mathrm{HNC}$ free flap patients.

\section{Limitations}

This study has some limitations. The study population is quite heterogenous since we included all HNC free flap patients into the analysis. Because the impact of free flap operations on nutritional delivery may vary significantly between the patients, the heterogenous study population may lead to different nutritional outcomes between different subgroups. However, by including all subgroups to the present analysis, our study design mimics actual surgical ward setting quite accurately. In the present study, it was not possible to analyze nutrition prescription rate since in our hospital, nutrition support is nurse driven and formal prescriptions 
are rarely done by the attending physician or dietician. The reasons why oral food delivery was not initiated during the first or second postoperative days in this cohort remains partly obscure. Initiation judgement of oral food delivery is ultimately done based by attending surgeon's evaluation. Problems in surgical area such as swelling, pain, poor tissue healing as well as attending surgeon's customs may postpone oral food initiation. However, to analyze this phenomenon in detail, it would require a study preferably in a prospective setting. The retrospective study design and a relatively short follow-up period of the study causes limitations when inspecting causal relationship between inadequate nutrition delivery and the rate of complications. However, based on our findings, it can be concluded that surgical complications are associated with inadequate nutrition delivery. Finally, the aim of the present study was to analyze nutrition adequacy and factors related to inadequate nutritional delivery, not to investigate complications associated underfeeding.

\section{Conclusion}

Underfeeding is common within HNC free flap patients during the immediate postoperative period. Early oral food intake was associated with increased nutrition delivery and nutrition support often failed to provide enough nutrition to cope with patient's energy demand. The results of the present study suggest that oral food intake is the most effective way to commence nutrition in HNC free flap patients postoperatively. Guidelines contemplating the issue promote early nutrition support for patients unable to ingest food [1, 10] but it seems that nutrition support alone is prone to be inadequate in this patient group. The findings of the present study should be taken into the account when contemplating the postoperative nutrition of $\mathrm{HNC}$ free flap patients.

Acknowledgments Open access funding provided by University of Oulu including Oulu University Hospital.

Funding This research did not receive any specific grant from funding agencies in the public, commercial, or not-for-profit sectors.

Data availability Not applicable.

\section{Compliance with ethical standards}

Conflict of interest The authors declare that they have no conflict of interest.

Ethics approval All procedures performed in studies involving human participants were in accordance with the ethical standards of the institutional research committee and with the 1964 Helsinki Declaration and its later amendments or comparable ethical standards. The study was approved by the hospital administration (208/2015 and 239/2016).

Informed consent Due to the retrospective cohort study design, no informed consent was obtained in accordance with Finnish law.

Open Access This article is licensed under a Creative Commons Attribution 4.0 International License, which permits use, sharing, adaptation, distribution and reproduction in any medium or format, as long as you give appropriate credit to the original author(s) and the source, provide a link to the Creative Commons licence, and indicate if changes were made. The images or other third party material in this article are included in the article's Creative Commons licence, unless indicated otherwise in a credit line to the material. If material is not included in the article's Creative Commons licence and your intended use is not permitted by statutory regulation or exceeds the permitted use, you will need to obtain permission directly from the copyright holder. To view a copy of this licence, visit http://creativecommons.org/licenses/by/4.0/.

\section{References}

1. Weimann A, Braga M, Carli F, Higashiguchi T, Hubner M, Klek $S$ et al (2017) ESPEN guideline: clinical nutrition in surgery. Clin Nutr 36(3):623-650

2. Stechmiller JK (2010) Understanding the role of nutrition and wound healing. Nutr Clin Pract 25(1):61-68

3. Stoppe C, Goetzenich A, Whitman G, Ohkuma R, Brown T, Hatzakorzian R et al (2017) Role of nutrition support in adult cardiac surgery: a consensus statement from an International Multidisciplinary Expert Group on Nutrition in Cardiac Surgery. Crit Care 21:10

4. Hill A, Nesterova E, Lomivorotov V, Efremov S, Goetzenich A, Benstoem C et al (2018) Current evidence about nutrition support in cardiac surgery patients-What do we know? Nutrients 10(5):597

5. Williams J, Wischmeyer P (2017) Assessment of perioperative nutrition practices and attitudes-a National Survey of Colorectal and GI surgical oncology programs. Am J Surg 213(6):1010-1018

6. Malafarina V, Reginster JY, Cabrerizo S et al (2018) Nutritional status and nutritional treatment are related to outcomes and mortality in older adults with hip fracture. Nutrients 10(5):555

7. Lahtinen S, Koivunen P, Ala-Kokko T, Kaarela O, Ohtonen P, Laurila P et al (2018) Complications and outcome after free flap surgery for cancer of the head and neck. Br J Oral Maxillofac Surg 56(8):684-691

8. McAuley D, Barry T, McConnell K, Smith J, Stenhouse J (2015) Early feeding after free flap reconstruction for oral cancer. Br J Oral Maxillofac Surg 53(7):618-620

9. Thomas WWT, Brant J, Chen J, Coblens O, Fischer JP, Newman JG et al (2018) Clinical factors associated with reoperation and prolonged length of stay in free tissue transfer to oncologic head and neck defects. JAMA Facial Plast Surg 20(2):154-159

10. Talwar B, Donnelly R, Skelly R, Donaldson M (2016) Nutritional management in head and neck cancer: United Kingdom National Multidisciplinary Guidelines. J Laryngol Otol 130(S2):S32-S40

11. Parhar HS, Durham JS, Anderson DW, Rush B, Prisman E (2020) The association between the Nutrition-Related index and morbidity following head and neck microsurgery. Laryngoscope 130(2):375-380

12. Shum J, Markiewicz MR, Park E, Bui T, Lubek J, Bell RB et al (2014) Low prealbumin level is a risk factor for microvascular free flap failure. J Oral Maxillofac Surg 72(1):169-177 
13. Timmermans AJ, Lansaat L, Kroon GV, Hamming-Vrieze O, Hilgers FJ, van den Brekel MW (2014) Early oral intake after total laryngectomy does not increase pharyngocutaneous fistulization. Eur Arch Otorhinolaryngol 271(2):353-358

14. Aires FT, Dedivitis RA, Petrarolha SM, Bernardo WM, Cernea CR, Brandao LG (2015) Early oral feeding after total laryngectomy: a systematic review. Head Neck 37(10):1532-1535

15. Suslu N, Sefik HA (2016) Early oral feeding after total laryngectomy: outcome of 602 patients in one cancer center. Auris Nasus Larynx 43(5):546-550

16. McClave SA, Taylor BE, Martindale RG, Warren MM, Johnson DR, Braunschweig C et al (2016) Guidelines for the provision and assessment of nutrition support therapy in the adult critically Ill patient: society of critical care medicine (SCCM) and American Society for parenteral and enteral nutrition (A.S.P.N.). JPEN J Parenter Enteral Nutr 40(2):159-211

17. Nurkkala JP, Kaakinen TI, Vakkala MA, Ala-Kokko TI, Liisanantti JH (2020) Nutrition deficit durinnce, predisposing factors and outcomes. Minerva Anestesiol 86(5):527-536

18. Lahtinen S, Koivunen P, Ala-Kokko T, Kaarela O, Laurila P, Liisanantti JH (2019) Swallowing-related quality of life after free flap surgery due to cancer of the head and neck. Eur Arch Otorhinolaryngol 276(3):821-826

19. Ohkoshi A, Ogawa T, Nakanome A, Ishida E, Ishii R, Kato K et al (2018) Predictors of chewing and swallowing disorders after surgery for locally advanced oral cancer with free flap reconstruction: a prospective, observational study. Surg Oncol 27(3):490-494

20. Lilja M, Markkanen-Leppanen M, Viitasalo S, Saarilahti K, Lindford A, Lassus P et al (2018) Olfactory and gustatory functions after free flap reconstruction and radiotherapy for oral and pharyngeal cancer: a prospective follow-up study. Eur Arch Otorhinolaryngol 275(4):959-966
21. Guidera AK, Kelly BN, Rigby P, MacKinnon CA, Tan ST (2013) Early oral intake after reconstruction with a free flap for cancer of the oral cavity. Br J Oral Maxillofac Surg 51(3):224-227

22. Rahman A, Agarwala R, Martin C, Nagpal D, Teitelbaum M, Heyland DK (2017) Nutrition therapy in critically Ill patients following cardiac surgery: defining and improving practice. JPEN J Parenter Enteral Nutr 41(7):1188-1194

23. Nurkkala J, Kaakinen T, Vakkala M, Ala-Kokko T, Liisanantti JH (2020) Factors associated with discrepancy between prescribed and administered enteral nutrition in general ICU. Eur J Clin Nutr 74(2):248-254

24. Fisher DF, Kondili D, Williams J, Hess DR, Bittner EA, Schmidt UH (2013) Tracheostomy tube change before day 7 is associated with earlier use of speaking valve and earlier oral intake. Respir Care 58(2):257-263

25. Bartella AK, Kamal M, Berman S, Steiner T, Frölich D, Hölzle F et al (2018) Role of swallowing function of tracheotomised patients in major head and neck cancer surgery. J Craniofac Surg 29(2):e122-e124

26. Goetz C, Burian NM, Weitz J, Wolff KD, Bissinger O (2019) Temporary tracheotomy in microvascular reconstruction in maxillofacial surgery: Benefit or threat? J Craniomaxillofac Surg 47(4):642-646

Publisher's Note Springer Nature remains neutral with regard to jurisdictional claims in published maps and institutional affiliations. 